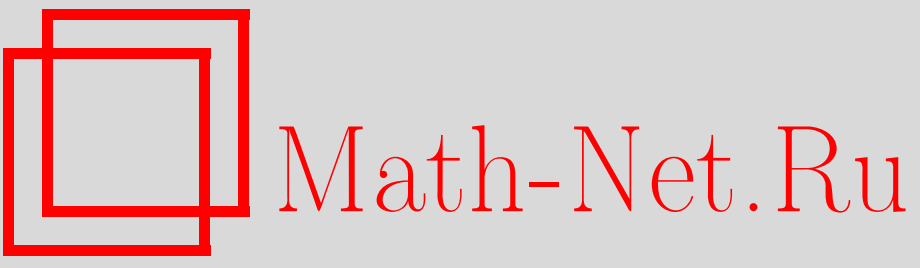

Е. Г. Скляренко, О когомологиях с носителями, УМН, 1996, том 51, выпуск 1, 167-168

DOI: https://doi.org/10.4213/rm936

Использование Общероссийского математического портала Math-Net.Ru подразумевает, что вы прочитали и согласны с пользовательским соглашением

http://www.mathnet.ru/rus/agreement

Параметры загрузки:

IP : 44.207 .124 .84

26 апреля 2023 г., $15: 12: 53$ 


\title{
О КОГОМОЛОГИЯХ С НОСИТЕЛЯМИ
}

\author{
Е. Г. СКляренко
}

Под семейством носителей принято понимать направленное по включению семейство $\Phi$ замкнутых множеств топологического пространства $X$ такое, что из $A \in \Phi$ и $B \subset A$ следует $B \in \Phi$ (п. 3.2 в [4]). Когомологии $H_{\Phi}^{*}(X ; \mathscr{G})$ пространства $X$ с коэффициентами в пучке $\mathscr{G}$ с носителями в $\Phi$ определяются как когомологии коцепного комплекса $\Gamma_{\Phi}\left(\mathscr{A}^{*}(\mathscr{G})\right)$, составленного всеми сечениями с носителями в $\Phi$ любой $\Phi$-ацикличной (например, вялой) резольвенты пучка $\mathscr{G}$.

Заметим, что для замкнутого множества $A$ и вялой резольвенты $\mathscr{C}^{*}(\mathscr{G})$ ограничение сечений $\Gamma\left(\mathscr{C}^{*}(\mathscr{G})\right)=\mathscr{C}^{*}(\mathscr{G})(X) \rightarrow \mathscr{C}^{*}(\mathscr{G})(X \backslash A)$ эпиморфно, а ядром служит комплекс $\Gamma_{A}\left(\mathscr{C}^{*}(\mathscr{G})\right)$ всех сечений с носителями в $A$. Таким образом, $H^{*}\left(\Gamma_{A}\left(\mathscr{C}^{*}(\mathscr{G})\right)\right)=H^{*}(X, X \backslash A ; \mathscr{G})$. Поскольку $\Gamma_{\Phi}\left(\mathscr{C}^{*}(\mathscr{G})\right)=\bigcup_{A \in \Phi} \Gamma_{A}\left(\mathscr{C}^{*}(\mathscr{G})\right)=\lim _{A \in \Phi}\left\{\Gamma_{A}\left(\mathscr{C}^{*}(\mathscr{G})\right)\right\}$, а точный функтор $\lim _{\longrightarrow}$ перестановочен с операцией $H^{*}$, имеем

ПРЕДЛОЖЕНИЕ 1. $H_{\Phi}^{*}(X ; \mathscr{G})=\lim _{A \in \Phi}\left\{H^{*}(X, X \backslash A ; \mathscr{G})\right\}$.

Смысл его, очевидно, в том, что когомологии с носителями в какой-то мере сводятся к обычным. Обсуждение этой меры и эпитета "обычные" - одна из целей заметки.

Семейство $\Phi$ называется окрестностным [7], если каждое $A \in \Phi$ имеет замкнутую окрестность: $A^{\prime} \in \Phi$. В [2] впервые были введены паракомпактифицирующие семейства - это окрестностные семейства замкнутых паракомпактных множеств. Пусть $\Psi$ - система множеств вида $X \backslash \operatorname{Int} A, A \in \Phi$.

СлеДСтвиЕ 2. Для окрестностных семейств $H_{\Phi}^{*}(X ; \mathscr{G})=\lim _{B \in \Psi}\left\{H^{*}(X, B ; \mathscr{G})\right\}$.

Для паракомпактифицирующих семейств этот факт был замечен еще А. Картаном. Вариант доказательства приведен в п. 3.10 в [4] (где вместо $H^{*}(X, B ; \mathscr{G})$ используются изоморфюые группы $\left.H^{*}\left(X ; \mathscr{G}_{X \backslash B}\right)\right)$.

Для замкнутого множества $F$ паракомпактного пространства $X$ и паракомпактифицирующего семейства $\Phi$ ограничение сечений $\Gamma_{\Phi}\left(\mathscr{C}^{*}(\mathscr{G})\right) \rightarrow \Gamma_{\Phi \cap F}\left(\mathscr{C}^{*}(\mathscr{G}) \mid F\right)$ эпиморфно (с учетом $\Phi$-мягкости вялых пучков, п. 3.3 в [4], см. предложение 9.3 главы 2 в [1]), а его ядро есть $\Gamma_{\Phi \mid(X \backslash F)}\left(\mathscr{C}^{*}(\mathscr{G})\right)$. В силу $\Phi$-ацикличности резольвенты $\mathscr{C}^{*}(\mathscr{G}) \mid F$ (вытекающей из утверждения (a) теоремы 3.5.5 главы II в [3] и предложения 9.8 главы 2 в [1]) указанные сечения на $F$ определяют $H_{\Phi \cap F}^{*}(F ; \mathscr{G} \mid F)=H_{\Phi}^{*}(F ; \mathscr{G})$, а указанное ядро - $H_{\Phi \mid(X \backslash F)}^{*}(X ; \mathscr{G})=H_{\Phi}^{*}(X \backslash F ; \mathscr{G})$.

ПреДЛОЖЕниЕ 3. Если $\Phi$ - паракомпактифицирующее семейство в паракомпактном пространстве $X$, то для $h \in H_{\Phi}^{*}(X ; \mathscr{G})$ найдутся $A \in \Phi$ и такое замкнутое множество $B \subset X$, что $X=A \cup B$ и образ $h$ в $H_{\Phi}^{*}(B ; \mathscr{G})$ равен нулю.

Это означает, очевидно, что элемент $h$ сосредоточен на $X \backslash B \subset A \in \Phi$.

Утверждение выполнено для множества $B \in \Psi$ (см. следствие), для которого $h$ содержится в образе $H^{*}(X, B ; \mathscr{G})$, и для $A \in \Phi$, являющегося замыканием $X \backslash B$.

Скажем (условно), что замкнутое множество $Y \subset X$ служит носителем некоторого $h \in$ $H_{\Phi}^{*}(X ; \mathscr{G})$, если существует замкнутое $B$, для которого $X=Y \cup B$ и ограничение $h$ на $B$ равно нулю. Таким способом определяются носители теории когомологий в [7] (см. также определение когомологий с компактными носителями в [6]).

ПрЕДЛОЖЕнИЕ 4. Если $\Phi$ - паракомпактифицирующее семейство в паракомпактном пространстве $X$, то носитель $Y$ любого $h \in H_{\Phi}^{*}(X ; \mathscr{G})$ содержит в себе носители $h$ из $\Phi$.

На самом деле, элемент $h$ с носителем $Y$ содержится в образе $H_{\Phi}^{*}(X, B ; \mathscr{G})=H_{\Phi}^{*}(X \backslash B ; \mathscr{G}) \rightarrow$ $H_{\Phi}^{*}(X ; \mathscr{G})$ и потому в силу предложения 1 представляется элементом из $H^{*}(X, X \backslash A ; \mathscr{G})$ для $A \subset X \backslash B \subset Y, A \in \Phi$.

Работа выполнена при финансовой поддержке Российского фонда фундаментальных исследований (грант № 94-01-00670), Международного научного фонда (грант № JH 2100) и Правительства России. 
ПредЛОжениЕ 5. Если $\Phi$ - паракомпактифицирующее семейство в паракомпактном $X$, то при $p>0$ для любой точки $x \in X$ имеем $\lim _{N}\left\{H_{\Phi}^{p}(N ; \mathscr{G})\right\}=0$, где $N$ - замкнутые окрестности $x$. Иньли словами, для $h \in H_{\Phi}^{p}(X ; \mathscr{G})$ u $x \in X$ найдется столь малая $N$, что ограничение $h$ на $N$ равно нулю.

Таким образом, как и в случае обычной теории $H^{*}$, элементы групп $H_{\Phi}^{*}$ должны занимать в пространстве $X$ определенньй "объем".

Для доказательства обозначим через $s$ определяюшее $h$ сечение $\mathscr{C}^{*}(\mathscr{G})$ с носителем $|s| \in \Phi$, $d s=0$. Если $x \notin|s|$, то $s$ обрашается в нуль в некоторой окрестности $N$ точки $x$, и ограничение $h$ на $N$ равно нулю. Пусть $x \in|s|$. Выберем $A \in \Phi$ такое, что $|s| \subset \operatorname{Int} A$. Росток $s$ в точке $x$ имеет вид $d s^{\prime}$ для сечения $s^{\prime}$ на некоторой замкнутой окрестности $N$. Если $N \subset A$, то $N \in \Phi$, и $h$ на $N$ равно нулю.

Картина резко меняется для непаракомпактифицирующих $\Phi$. Для пар $(A, N)$, где $A \in \Phi$ и $N$ - замкнутая окрестность $x$, полагаем $(A, N)<\left(A^{\prime}, N^{\prime}\right)$, если $A \subset A^{\prime}$ и $N^{\prime} \subset N$. Легко видеть, что ${\underset{\lim }{\longrightarrow} \in N}_{N}\left\{H_{\Phi}^{p}(N ; \mathscr{G})\right\}=\lim _{(A, N)}\left\{H^{p}(N, N \backslash A ; \mathscr{G})\right\}$. В тех случаях, когда $x \notin \operatorname{Int} A$ для всех $A \in \Phi$, обращение в нуль указанного предела маловероятно.

Пусть, например, $X$ - (нульмерное) пространство рациональных точек интервала $(0,1)$, $\mathscr{G}=\mathbb{Z}$ - целые числа и $\Phi=c$ - непаракомпактифицирующее семейство компактных множеств (паракомпакифицируемость $c$ эквивалентна локальной компактности пространства). Для $C \in c$ рассмотрим точную последовательность $\cdots \rightarrow H^{0}(X ; \mathbb{Z}) \stackrel{i}{\longrightarrow} H^{0}(X \backslash C ; \mathbb{Z}) \rightarrow$ $H^{1}(X, X \backslash C ; \mathbb{Z}) \rightarrow 0$. Обе первые группы - это группы локально постоянных функций, принимающих значения в $\mathbb{Z}$. Поскольку в первой из групп они постоянны вблизи точек $x \in C$, отображение $i$ - включение, и $H^{1}(X, X \backslash C ; \mathbb{Z}) \neq 0$. Сопоставляя две точные последовательности, отвечающие включению $C \subset C^{\prime}$, заключаем, что отображение $H^{1}(X, X \backslash C ; \mathbb{Z}) \rightarrow H^{1}\left(X, X \backslash C^{\prime} ; \mathbb{Z}\right)$ - мономорфизм. В силу предложения $1 H_{c}^{1}(X ; \mathbb{Z}) \neq 0$.

Отличный от нуля элемент $h \in H_{c}^{1}(X ; \mathbb{Z})$ содержится в некоторой группе $H^{1}(X, X \backslash C ; \mathbb{Z})$ и потому представляется локально постоянной функцией на $X \backslash C$, не постоянной вблизи некоторой точки $x \in C$ (отличной от константы в сколь угодно малом интервале вокруг $x$ ). Это свойство сохраняется при ограничениях на множества $X \backslash C^{\prime}$ для любых содержащих $C$ множеств $C^{\prime} \in c$. Это означает (ср. с предложением 5), что ограничения $h$ на сколь угодно малые окрестности точки $x$ отличны от нуля.

Приведенный пример нельзя считать нетипичным, поскольку отличие от нуля $H^{p}(X, U ; \mathscr{G})$ для открытых $U$ в размерности $p=\operatorname{dim} \varphi X+1-$ явление довольно частое (см. $\S 5$ в [5]). Представляет интерес даже тот частный случай, когда $\Phi=\{x\}-$ одна точка. В этом случае $H_{\Phi}^{p}(X ; \mathscr{G})=H^{p}(X, X \backslash x ; \mathscr{G})=H_{x}^{p}(\mathscr{G})$ - локальная группа когомологий, изоморфная пределу $\lim \left\{H^{p-1}(U \backslash x ; \mathscr{G})\right\}$ по окрестностям $U$ точки $x(\S 1$ в [5]). Ясно, что и для этих групп утверждение предложения 5 не имеет места. Существует (локально) стягиваемый двумерный компакт $X$ (для таких пространств, кстати, пучковые когомологии изоморфны сингулярным, $\S 1$ главы 3 в [1]) с такой точкой $x \in X$, что $X \backslash x$-полиэдр, но $H^{3}(X, X \backslash x ; \mathbb{Z}) \neq 0$ (пример 4.6 в [5]).

Таким образом, когомологии $H_{\Phi}^{*}$ сводятся к "обычным" только для паракомпактифицирующих (или окрестностных) семейств $\Phi$, без этого же условия они имеют другую природу, оказываясь в большой степени зависимыми от локальной структуры пространств.

\section{СПИСОК ЛИТЕРАТУРЫ}

[1] Бредон Г. Э. Теория пучков. М.: Наука, 1988. [2] Cartan Н. Cohomologie des groups, suite spectral, faisceaux. Séminaire. Paris: Ecole Norm. Super, 1950-1951. [3] Годеман P. Алгебраическая топология и теория пучков. М.: ИЛ, 1961. [4] Grothendieck A. // Tôhoku Math. J. 1957. V. 9. Р. 119-211. [5] Скляренко Е. Г. // Изв. АН СССР. Сер. матем. 1980. Т. 44. № 6. C. 1417-1433. [6] Spanier E. // Contemporary Math. 1982. V. 12. P. 315-329. [7] Spanier E. // Pacif. J. Math. 1986. V. 123. № 2. P. 447-464. 